\title{
The effect of gravity on coral morphology
}

\author{
Efrat Meroz, Itzchak Brickner, Yossi Loya, Adi Peretzman-Shemer \\ and Micha Ilan
}

\author{
Department of Zoology, Tel Aviv University, Tel Aviv 69978, Israel
}

\begin{abstract}
Coral morphological variability reflects either genetic differences or environmentally induced phenotypic plasticity. We present two coral species that sense gravity and accordingly alter their morphology, as characterized by their slenderness (height to diameter) ratio (SR). We experimentally altered the direction (and intensity) of the gravitational resultant force acting along or perpendicular to the main body axis of coral polyps. We also manipulated light direction, in order to uncouple gravity and light effects on coral development. In the experiments, vertically growing polyps had significantly higher SR than their horizontal siblings even when grown in a centrifuge (experiencing different resultant gravitational forces in proximal and distal positions). Lowest SR was in horizontal side-illuminated polyps, and highest in vertical top-illuminated polyps. Adult colonies in situ showed the same pattern. Gravitational intensity also affected polyp growth form. However, polyp volume, dry skeleton weight and density in the various centrifuge positions, and in aquaria experiments, did not differ significantly. This reflects the coral's ability to sense altered gravity direction and intensity, and to react by changing the development pattern of their body morphology, but not the amount of skeleton deposited.
\end{abstract}

Keywords: developmental plasticity; light; pattern formation; phenotypic plasticity; slenderness ratio; Stylophora pistillata

\section{INTRODUCTION}

When a species encounters heterogeneous environments within its range, it is unlikely that any single phenotype will confer high fitness in all situations (Via et al. 1995). An organism's potential to produce a range of different, relatively fit, phenotypes in several environments gives it ecological and evolutionary advantages (DeWitt et al. 1998). Individual ability to alter physiology and/or morphology in response to changing environmental conditions is termed phenotypic plasticity and such ability is particularly important for sessile organisms, which cannot retreat from changing conditions (Bruno \& Edmunds 1997). However, morphological variation that is common among clonal organisms, including scleractinian corals (Willis 1985; Bruno \& Edmunds 1997), is the result of either genetic differences between individuals (or populations) (Ayre \& Willis 1988), or environmentally induced phenotypic plasticity (Bruno \& Edmunds 1997).

Earlier studies using transplantation experiments investigated a possible existence of phenotypic plasticity in coral morphology or skeletal strength at different depths (and hence related factors, such as light (Willis 1985)), sedimentation rates (Foster 1980) and hydrodynamic stress (Jokiel 1978; Helmuth \& Sebens 1993). Although ubiquitous, the gravitational effect on pattern formation of sessile marine invertebrates is virtually unstudied. Gravity is probably particularly important for certain situations or taxa, e.g. on steep slopes, overhanging roofs or vertical walls, encrusting versus erect growth forms, etc.

Gravity affects biological systems (Anon. 1984; Schmitt et al. 1999), including organism morphology. An altered direction of gravity influences the internal cell

${ }^{*}$ Author for correspondence (milan@post.tau.ac.il). arrangement, causing general shape change by a new pattern formation (Tabony \& Job 1992; Moore et al. 1996). Gravitational influence is not limited to the mechanical relationships between organism parts and extends to the biochemical and even molecular levels of metabolic control (Moore et al. 1996). Starting from Cnidaria, marine mobile and sessile organisms of all phyla possess gravity sensors (Lowenstam \& Weiner 1989). In addition to specialized sensory cells in animals and plants, altered gravitational acceleration also affects cells with no obvious structural specialization to sense gravity, implying some direct effect of gravity on cellular function (Moore $e t$ al. 1996; Ingber 1999). For example, gravity variations influence osteoblast morphology, inducing cell shape changes (Guignandon et al. 1995). Gravity also influences organism orientation, plankton vertical migration and larval settlement patterns of sessile marine organisms (Ishay \& Sadeh 1975; Pires \& Woollacott 1983; Macquart-Moulin 1999).

The present study examined the influence of gravity on the growth form of young polyps of two common Red Sea scleractinian coral species. Coral pattern formation was examined with respect to the orientation of the polyp longitudinal axis, i.e. horizontal or vertical (figure 1), and the influence of different gravity forces was evaluated. In addition, growth forms of adult colonies growing in situ on horizontal versus vertical substrate were compared.

\section{MATERIAL AND METHODS}

The field site was the reef near Elat (Red Sea). Planulae of the stony corals Stylophora pistillata and Seriatopora hystrix were collected in situ using plankton net traps placed over colonies in the late afternoon and removed after planulation: $S$. pistillata at night (21.00-23.00) and S. hystrix at early morning (02.0004.00). After removal, the nets were closed and kept in ziplock 


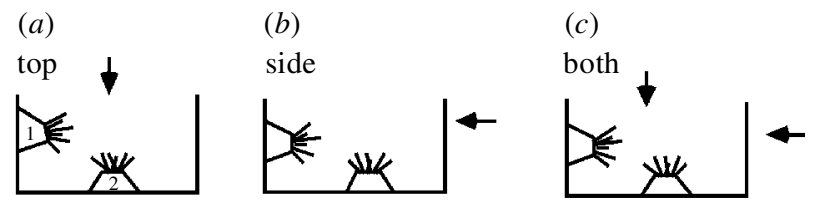

(d)

(e)
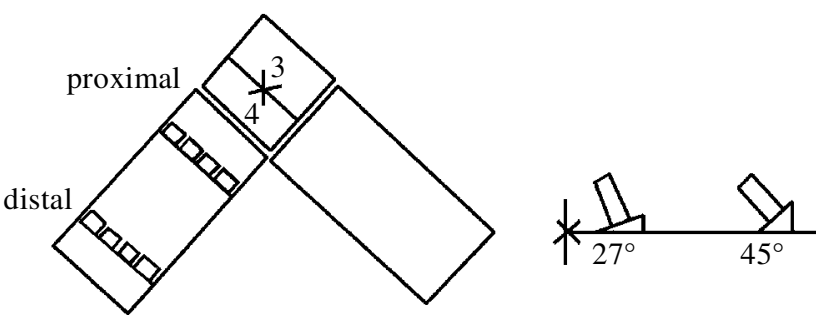

Figure 1. Experimental set-up: $(a-c)$ aquaria experiments, $(d, e)$ centrifuge experiments. Arrows indicate direction of illumination source. (a) Top illuminated (1, horizontal polyps; 2 , vertical polyps), (b) side illuminated, and (c) top and side illuminated. $(d)$ Top view of two of the centrifuge's four arms. 3 and 4 indicate two posts of rotating control. (e) Side view of one arm of the centrifuge. Proximal location with a slope of $27^{\circ}$ and distal location with a slope of $45^{\circ}$.

bags full of seawater, and then transferred to Petri dishes in the laboratory for settlement.

\section{(a) Aquaria experiments}

Petri dishes with settled planulae were placed in aerated aquaria with filtered seawater (that has been passed through a sieve of hole size $0.45 \mu \mathrm{m}$ ), fixed in either a horizontal or a vertical direction. Aquaria were either top or side illuminated, or both for $18 \mathrm{~h}$ every day (figure 1). Aquaria water was replaced once a week. Algal growth around young polyps was cleaned twice a week to prevent substrate limitation or competition. Onemonth-old polyps were removed from the aquaria and their skeleton diameter and perpendicular height were measured by a dissecting microscope micrometer. These parameters were used to calculate each polyp's volume and their slenderness (height to diameter) ratio (SR). Volume and SR were used to compare morphological plasticity between polyps grown in horizontal versus vertical orientations. Following height and diameter measurement, polyps were dried at $70^{\circ} \mathrm{C}$ for $24 \mathrm{~h}$ to examine their skeleton dry weight and density (weight/volume).

\section{(b) Centrifuge experiments}

To examine if intensity of gravity also affects coral morphology, planulae were grown in vials put in a four-constantarmed centrifuge (Ishay \& Sadeh 1975). Each vial with $40 \mathrm{ml}$ of filtered seawater contained 20 Stylophora pistillata planulae. Each arm of the centrifuge held vials in both proximal and distal positions (figure $1 d, e$ ), and it worked continuously, illuminated from above for $18 \mathrm{~h}$ every day, for a total of 30 days. At $25 \mathrm{rpm}$ rotation, the resultant gravitational force of $11.07 \mathrm{~m} \mathrm{~s}^{-2}$ acted on the proximal position, and $13.9 \mathrm{~m} \mathrm{~s}^{-2}$ on the distal position. The angle of the resultant force acting on each position is given by: $\tan \varnothing=(2 \pi f)^{2} r / g=4 \pi^{2}(25 / 60)^{2} r / 980$, where $2 \pi f$ is the angular frequency, $r$ is the radial distance from the centre, and the constant of gravity $g$ is $9.8 \mathrm{~m} \mathrm{~s}^{-2}$. Corresponding angles for the proximal and distal positions were $27^{\circ}$ and $45^{\circ}$, respectively. In the proximal and distal positions, the vials were attached on a slope of $27^{\circ}$ and $45^{\circ}$, respectively, so that when the centrifuge was rotating, the water surface was parallel to these slopes (figure 1e). The resultant force was thus at $90^{\circ}$ to the vial base, enabling the horizontal and vertical positions to remain as though without a centrifuge (the vial base was considered as the horizontal substrate and the vial wall as the vertical substrate). Two controls were used in this experiment: a rotating control, positioned $5 \mathrm{~cm}$ from the centre of the centrifuge, with a gravitational force of $9.806 \mathrm{~m} \mathrm{~s}^{-2}$ (figure $1 d$ ); and a stationary control, placed adjacent to the centrifuge, with a gravitational force of $9.8 \mathrm{~m} \mathrm{~s}^{-2}$ (figure $1 d$ ).

\section{(c) Field survey}

To compare the laboratory results with the natural situation, diameter and perpendicular height of adult $S$. pistillata were measured in colonies grown on the reef in a vertical or horizontal orientation. Colony SR was compared between corals grown at 5 and $10 \mathrm{~m}$ depths, at horizontal or vertical orientations.

\section{RESULTS}

Taking into account the relative available area for settlement in each orientation, planulae of both coral species showed no settlement preference at horizontal or vertical orientation ( $p \gg 0.05$ ). High percentages (95\%) of polyps survived until the termination of the experiments (S. pistillata, $n=207 ; S$. hystrix, $n=107$ ). Stylophora pistillata polyps were grown for four to five weeks, developing into small colonies (five to six polyps), S. hystrix grew more slowly, producing only solitary polyps.

The volume of both species' polyps in different orientations (horizontal versus vertical) did not differ significantly. The direction of illumination (top, side and both; figure 1) had no significant effect on the volume of S. pistillata polyps, but in $S$. hystrix, top-illuminated polyps had a significantly larger volume than side-illuminated ones. Examination of polyp morphology, however, revealed that vertical polyps of both species had significantly higher SR than horizontal polyps (figure 2). Polyps of both species showed significantly different SR under different directions of illumination. Top-illuminated polyps had significantly higher SR compared with sideilluminated polyps, regardless of orientation. In both species, side-illuminated horizontal polyps had the lowest SR, and top-illuminated vertical polyps had the highest SR. Interactions between orientation and direction of illumination were non-significant for both species.

Polyps grown in different positions on the four-armed centrifuge (figure 1) exhibited the pattern described above for aquaria-raised polyps. In each position (distal, proximal, rotating control and stationary control), mean SR of vertical polyps was significantly higher $(p=0.01)$ than that of horizontal polyps (figure 3 ). In each position, polyp volume, skeleton dry weight and skeleton density did not differ significantly. These polyps' SRs, however, differed significantly $(p<0.0001)$, with the lowest SR for distal polyps regardless of orientation (a posteriori Scheffe analysis, $p \ll 0.01$; figure 3 ). Polyps' SRs in the proximal position did not differ significantly from the controls.

As SR values of $40 \mathrm{~S}$. pistillata colonies that were measured in the reef did not differ significantly between depths, they were combined for each orientation. Between orien- 

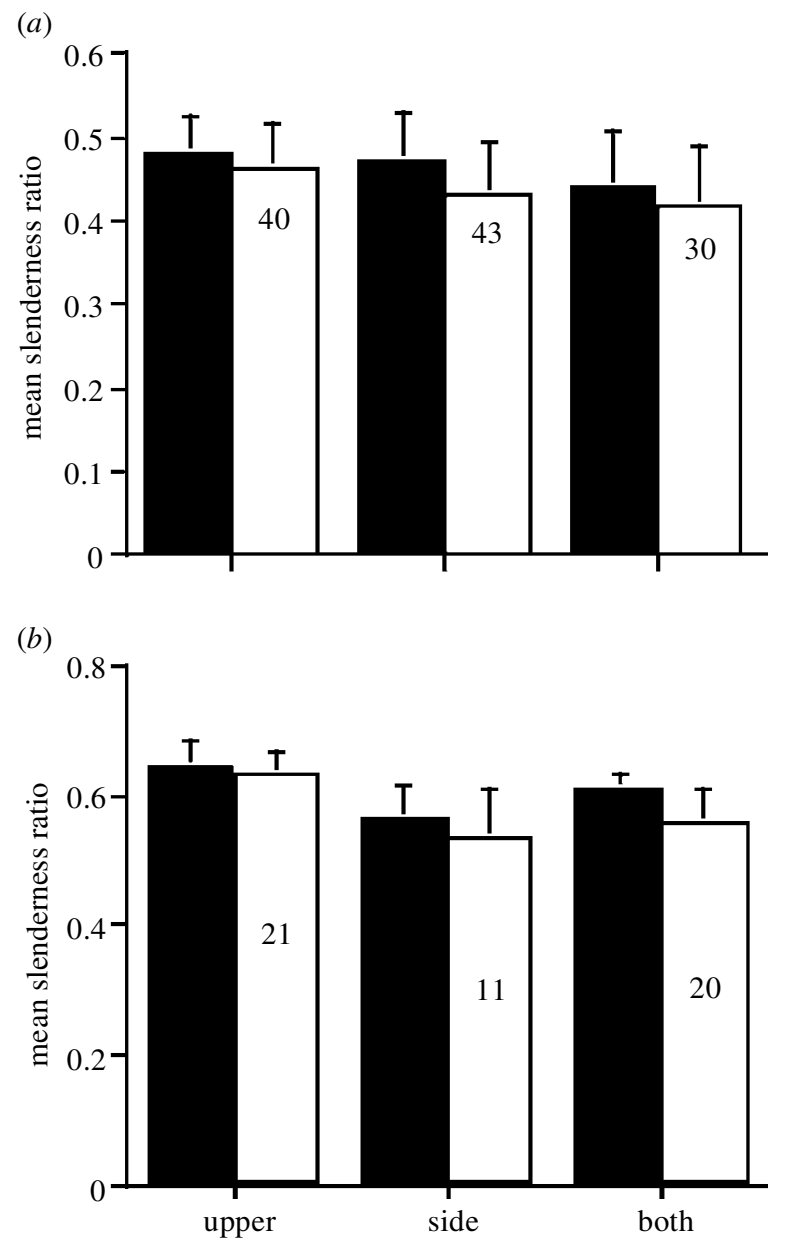

direction of illumination

Figure 2. Polyps' mean slenderness (height to diameter) ratio under different directions of illumination (+ s.d.) in aquarium experiments. (a) Polyps of Stylophora pistillata (vertical versus horizontal polyps, ANOVA, $p<0.005$ ). (b) Polyps of Seriatopora hystrix (vertical versus horizontal polyps, two-way ANOVA and a posteriori tests using STATVIEW v. 4.05, $p<0.001)$. Black bars, vertical orientation; white bars, horizontal orientation. Numbers on bars are number of polyps.

tations, however, vertical colonies had a significantly higher SR than horizontal colonies (figure 4).

\section{DISCUSSION}

Orientation of the main body axis of corals affected the growth form of both the primary polyp and the whole colony. Aquaria and centrifuge experiments, confirmed by field survey, showed that SR was always higher for polyps and colonies in a vertical compared with horizontal orientation. Polyps' volume, dry skeleton weight or density, in both orientations (in aquaria or centrifuge), however, did not differ significantly, although growth form (as expressed by SR) differed significantly. This situation of two polyps with similar volumes and skeleton weights but different $S R$, indicates that their growth (calcification) rate is equal but their growth form differs, i.e. the proportion of height to diameter changed according to polyp orientation. What controls such change in pattern formation?

By adjusting shape or structure, sessile marine organ-

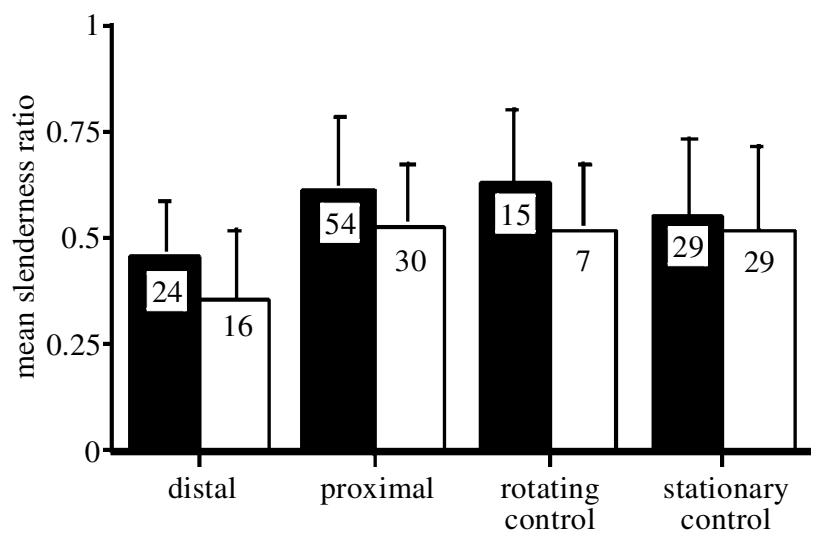

position on the centrifuge arm

Figure 3. Mean slenderness (height to diameter) ratio (SR) (+ s.d.) of Stylophora pistillata polyps after one month in the centrifuge. Black bars, vertical polyps; white bars, horizontal polyps. Numbers on bars are number of polyps. Differences in SR were compared by a non-parametric Mann-Whitney test.

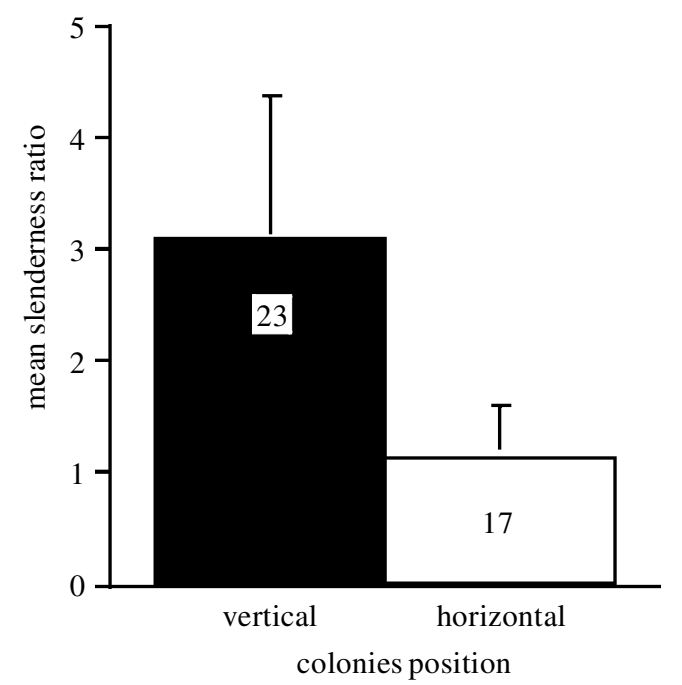

Figure 4. Mean slenderness (height to diameter) ratio (SR) (+ s.d.) of adult Stylophora pistillata colonies on the reef. Black bar, vertical colonies; white bar, horizontal colonies. SR of vertical versus horizontal polyps differs significantly (Mann-Whitney, $p<0.0001$ ). Numbers on bars are number of colonies.

isms respond to a given set of environmental parameters (Denny 1994). Physical forces such as water flow (Denny 1994) and sedimentation (Rogers 1990) may result in organisms with altered structures. A body's SR value affects flow, thus determining the particle fluxes encountered by benthic organisms (Abelson et al. 1993). In the experiments reported here, however, no water flow existed in the aquaria or the centrifuge. We therefore suggest that maybe in these experiments the short and wide shape of horizontal polyps (smaller SR) provides them with a better attachment to a vertical substrate against gravity that acts to detach them; whereas vertical organisms grow in a high, narrow shape (high SR). Moreover, in nature, horizontal corals face the risk of detachment (or breakage) by the vector force that combines both drag and gravity, whereas in vertical corals, gravity may even act against detachment 
by drag. But do the polyps sense gravity, or some other variable, such as illumination?

Organisms may use natural illumination from above to sense their orientation. However, in our experiments, vertical polyps had a significantly higher SR than horizontal polyps, regardless of direction of illumination (figure $2 a, b$ ). Had the illumination been the only factor to determine polyps' SR, side-illuminated horizontal polyps would have been expected to have the same mean SR as top-illuminated vertical polyps. Light intensity affects coral calcification, having lower rates (and therefore lower skeleton growth rate) with diminishing light intensity on a reef (Huston 1985). We therefore suggest that the coral growth pattern observed here reminds us of the pattern formation demonstrated by cells that remodel astronauts' bones. These cells sense gravity-caused changes in their local environment and react by secreting a new matrix in one direction instead of another (Ingber 1999). Such sensing of altered gravity direction and intensity can be achieved even on a cellular or molecular basis without a need for a specific organ or cell (Tabony \& Job 1992; Papaseit et al. 2000).

Planulae of both coral species settle and survive without preferred orientation. Gravity direction in relation to that of the body axis, however, induces different shapes for corals growing in different orientations. This explains why $S$. pistillata colonies from different orientations in the reef had the same SR pattern as corals in the aquaria experiments. It is therefore not surprising that in both the laboratory and nature, the highest ratio in both species was for top-illuminated vertical polyps (the most common combination found in nature) and the smallest was for side-illuminated horizontal polyps. Furthermore, this phenomenon probably has a wider distribution (e.g. massive corals that grow in a much flatter morphology on vertical natural walls or on sunken ships).

Intensity of gravity also influenced polyp growth form (figure 3). Polyps in the centrifuge distal position had significantly lower SR than those in the proximal position (the former experiencing higher gravitational resultant force). This resembles hypergravity influence on other calcification processes (e.g. calcification of statolith; Pedrozo et al. 1996).

The experiments and reef survey results show the importance of coral orientation in determining their pattern formation. We suggest that corals sense gravitational direction and intensity, and react by altering pattern formation. These capabilities are of utmost importance as gravitation is an omnipresent force, does not change and therefore is reliable for the organism's assessment of the various factors correlated with it.

We are grateful to J. Ishay for the use of his centrifuge and M. Hay, T. P. Hughes, J. R. Pawlik and B. Rinkevich, for critical reading of earlier versions of this manuscript.

\section{REFERENCES}

Abelson, A., Miloh, T. \& Loya, Y. 1993 Flow patterns induced by substrata and body morphologies of benthic organisms, and their roles in determining availability of food particles. Limnol. Oceanogr. 38, 1116-1124.
Anon 1984 Spacelab I reports. Science 225, 205-235.

Ayre, D. J. \& Willis, B. L. 1988 Population structure in the coral Pavona cactus: clonal genotypes show little phenotypic plasticity. Mar. Biol. 99, 495-505.

Bruno, J. F. \& Edmunds, P. J. 1997 Clonal variation for phenotypic plasticity in the coral Madracis mirabilis. Ecology 78, 2177-2190.

Denny, M. W. 1994 Roles of hydrodynamics in the study of life on wave-swept shores. In Ecological morphology (ed. P. C. Wainwright \& S. M. Reilly), pp. 169-204. University of Chicago Press.

DeWitt, T. J., Sih, A. \& Wilson, D. S. 1998 Cost and limits of phenotypic plasticity. Trends Ecol. Evol. 13, 77-81.

Foster, A. B. 1980 Environmental variation in skeletal morphology within the Caribbean reef corals Montastrea annularis and Siderastrea siderea. Bull. Mar. Sci. 30, 678-709.

Guignandon, A., Vico, L., Alexandre, C. \& Lafage-Proust, M. H. 1995 Shape changes of osteoblastic cells under gravitational variations during parabolic flight-relationship with PGE2 synthesis. Cell. Struct. Funct. 20, 369-375.

Helmuth, B. \& Sebens, K. 1993 The influence of colony morphology and orientation to flow on particle capture by the scleractinian coral Agaricia agaricites (Linnaeus). F. Exp. Mar. Biol. Ecol. 165, 251-278.

Huston, M. 1985 Variation in coral growth rates with depth at Discovery Bay, Jamaica. Coral Reefs 4, 19-25.

Ingber, D. 1999 How cells (might) sense microgravity. FASEB 7. 13(Suppl.), S3-S15.

Ishay, J. \& Sadeh, D. 1975 Direction finding by hornets under gravitational and centrifugal forces. Science 190, 802-804.

Jokiel, P. L. 1978 Effect of water motion on reef corals. F. Exp. Mar. Biol. Ecol. 35, 87-97.

Lowenstam, H. A. \& Weiner, S. 1989 On biomineralization. Oxford University Press.

Macquart-Moulin, C. 1999 Diel vertical migration and endogenous swimming rhythm in Asdterope mariae (Baird) and Philomedes interpuncta (Baird) (Crustacea Ostracoda Cypridinidae). F. Plankt. Res. 21, 1891-1910.

Moore, D., Hock, B., Greening, J. P., Kern, V. D., Frazer, L. N. \& Monzer, J. 1996 Centenary review-gravimorphogenesis in agarics. Mycol. Res. 100, 257-273.

Papaseit, C., Pochon, N. \& Tabony, J. 2000 Microtubule selforganization is gravity dependent. Proc. Natl Acad. Sci. USA 97, 8364-8368.

Pedrozo, H. A., Schwartz, Z., Luther, M., Dean, D. D., Boyan, B. D. \& Wiederhold, M. L. 1996 A mechanism of adaptation to hypergravity in the statocyst of Aplysia californica. Hear. Res. 102, 51-62.

Pires, A. \& Woollacott, R. M. 1983 A direct and active influence of gravity on the behavior of a marine invertebrate larva. Science 220, 731-733.

Rogers, C. S. 1990 Responses of coral reefs and reef organisms to sedimentation. Mar. Ecol. Prog. Ser. 62, 185-202.

Schmitt, D., Lapiere, C. \& Bouillon, R. (eds). 1999 Cell and molecular biology research in space. FASEB f. 13(Suppl.).

Tabony, J. \& Job, B. 1992 Gravitational symmetry breaking in microtubular dissipative structures. Proc. Natl Acad. Sci. USA 89, 6948-6952.

Via, S., Gomulkiewicz, R., De Jong, G., Scheiner, S. M., Schlichting, C. D. \& Van Tienderen, P. H. 1995 Adaptive phenotypic plasticity: consensus and controversy. Trends Ecol. Evol. 10, 212-217.

Willis, B. L. 1985 Phenotypic plasticity versus phenotypic stability in the reef corals Turbinaria mesentarina and Pavona cactus. In Proc. 5th Int. Coral Reef Congr., vol. 4 (ed. C. Gabrie \& B. Salvat), pp. 107-112. Moorea, French Polynesia: Antenne Museum-EPHE. 